\title{
Phenotypic and genotypic variant of MDR-Mycobacterium tuberculosis multiple isolates in the same tuberculosis episode, Rio de Janeiro, Brazil
}

\author{
M.K.N. Andrade ${ }^{1}$, S.M.A. Machado ${ }^{2}$, M.L. Leite ${ }^{1}$ and M.H.F. Saad ${ }^{2}$ \\ ${ }^{1}$ Hospital Municipal Raphael de Paula Souza, Instituto de Doenças do Tórax, Universidade Federal do \\ Rio de Janeiro, Rio de Janeiro, RJ, Brasil \\ 2Laboratório de Microbiologia Celular, Instituto Oswaldo Cruz, Fundação Oswaldo Cruz, Rio de Janeiro, \\ RJ, Brasil
}

Correspondence to: M.H.F. Saad, Laboratório de Microbiologia Celular, Instituto Oswaldo Cruz, Fiocruz, Av. Brasil, 4365, 21040-360 Rio de Janeiro, RJ, Brasil

Fax: +55-21-2270-9997. E-mail: saad@ioc.fiocruz.br

\begin{abstract}
Assuming that the IS6110-restriction fragment length polymorphism (RFLP) changes at a constant rate of 3.2 years, this methodology was applied to demonstrate, for the first time, variant patterns of Mycobacterium tuberculosis (MTB) in multiple isolates obtained at short time intervals from sputum and blood of an $\mathrm{HIV}^{+}$patient with multiple admissions to the Emergency Room and to the multidrug-resistant tuberculosis (MDR-TB) Reference Center of a secondary-care hospital in Rio de Janeiro, Brazil. In sputum, the IS6110-RFLP appeared in isolates with two variant patterns with 10 and 13 IS6110 copies. However, blood presented only the pattern corresponding to 10 copies, suggesting compartmentalization. With regard to the exact match of 10 of 13 bands, this may be a subpopulation with the same clonal origin and this may be related to the IS6110 transposition. A susceptibility test demonstrated an MDR profile (INHR $, R I F^{R}, S M^{R}$, and $\left.E M B^{R}\right)$, with the sputum isolate also exhibiting EMB $(R$ $=$ resistant; $\mathrm{S}=$ sensitive). A gene mutation confirmed resistance only to streptomycin. There was agreement between the results of the phenotypic test and the clinical response to MDR-TB treatment, suggesting serious implications with regard to treatment administration based exclusively on molecular methods, and calling attention to the fact that more effective control strategies against the emergence of MDR strains must be implemented by the TB control program to prevent transmission of MDR-MTB strains at health facilities in areas highly endemic for TB.
\end{abstract}

Key words: Tuberculosis; Mycobacterium tuberculosis; TB-MDR; TB-RFLP; Gene mutation; Fingerprint

Research supported by FAPERJ (\#E-26/111.657/08-9) and CNPq (\#33.654.831/0001-36).

Received July 25, 2008. Accepted January 6, 2009

\section{Introduction}

Traditionally, it used to be assumed that each tuberculosis (TB) patient was infected with only a single strain of Mycobacterium tuberculosis(MTB). Therefore, several MTB strains within the same patient were ignored until simultaneous infection with two or more strains was described (1). Brazil has a high TB incidence, the State of Rio de Janeiro reporting up to $90 / 100,000$ inhabitants in some geographic areas (2). To our knowledge, no report on multidrug resistant (MDR) polyclonal infection or diversity in the M. tuberculosis progeny during the same TB episode has ever been genetically confirmed in Brazil. We report here a patient with multiple admissions to various public health facilities, from whom genotypic variants were obtained in multiple MDR-MTB isolates as determined by the phenotypic susceptibility test and as suspected on the basis of clinical evaluation. However, considering gene mutation in 
selected genes such as inh $A, r p o B, r p s L$, and $e m b B$, which are most frequently associated with resistance to isoniazid (INH), rifampicin (RIF), streptomycin (SM), and ethambutol (EMB), there was discordance since wild-type profile was obtained for most of them.

\section{Case description and laboratory procedures}

On September 1st, 2003, a 49-year-old patient was admitted for the second time to Hospital Raphael de Paula Souza (HRPS), a secondary-care hospital with 31 beds and a Reference Center for Acquired Immunodeficiency Syndrome and TB in Rio de Janeiro city, southeast Brazil. Patient history classified him as homeless, homosexual, alcoholic, seropositive for human immunodeficiency virus (HIV) since May 2003 and treated 20 years previously for TB for 6 months. Earlier, the patient had been admitted to the emergency room from February to May 2003 due to a right shin fracture, and later he was transferred to HRPS due to fever, diarrhea, vomiting, and abdominal pain in order to investigate intestinal TB. Treatment for TB (probable diagnosis) was initiated with INH, RIF and pirazinamide (PZA) but was discontinued 15 days later because of hepatotoxic side effects and non-microbiological confirmation of TB. After hospital discharge, the patient did not comply with antiretroviral therapy, lived in a public shelter and on September 1st presented general weakness, weight loss and fever, diffuse abdominal pain, diarrhea with blood, vomiting, and first-degree malnutrition. The patient did not complain of respiratory insufficiency but had oral candidiasis. A chest $\mathrm{X}$-ray revealed a volumetric decrease in the left lung with upper pleural opacity, interstitial infiltration in the lower left lobe and pulmonary cavitation in the upper right lobe. Abdominal ultrasonography was normal, gastrointestinal tract endoscopy displayed a slight antral gastritis and a hematological exam detected anemia and lymphopenia. The patient's clinical specimens were negative for histoplasmosis, aspergillosis and paracoccidiosis according to a gel immunodiffusion test, and the blood culture was negative for enterobacteria and cocci. Treatment with ciprofloxacin was started for the gastrointestinal tract infection. For TB investigation, three samples of blood (9/3/2003) and four of sputum (9/16/2003; 9/19/2003) were obtained for bacterioscopy and culture. Mycobacterial isolates were identified by standard biochemical methods. Drug susceptibility testing (DST) was performed for INH, RIF, SM, and EMB (BACTEC 460 System, Becton Dickinson, USA). DST was repeated adopting the LowensteinJensen proportion method at the Mycobacteriology Laboratory, HU/UFRJ (3). The test was carried out blindly at various institutions. Regions of the inhA/katG, rpoB, rpsL, and $e m b B$ genes, in which mutations have been most frequently associated with resistance to INH, RIF, SM, and $\mathrm{EMB}$, respectively, were amplified by polymerase chain reaction under previously reported conditions and using previously reported oligonucleotide primers (4-8). Amplimers were evaluated for mutations using automated DNA sequence analysis performed on an $A B I 373$ sequencing apparatus according to the protocol supplied by the manufacturer using the Big Dye ${ }^{\mathrm{TM}}$ Terminator Cycle Sequencing Ready Reaction Kit (PE Applied Biosystems, USA). MTB-DNA isolates were typed by the international standardized IS6110-restriction fragment length polymorphism (RFLP) method as described previously, with minor modifications $(9,10)$.

\section{Patient follow-up, Results and Discussion}

On September 16th, one of the sputum samples was positive for acid-fast bacilli. Consequently, inpatient TB treatment was the first priority with ofloxacin, SM and EMB instead of RIF, INH, and PZA due to the presence of altered hepatic enzymes. Blood and sputum culture yielded growth in three and four tubes, respectively. Blood and sputum strains resulted in MDR patterns for INH, RIF, SM and $E M B$, with both $E M B^{S}$ and $E M B^{R}$ profiles for sputum strains $(R=$ resistant; $S=$ sensitive). Subsequently, an alternative treatment scheme with amikacine, EMB, clofazimine, quinolone, and terizidone was administered (11). There was clinical and radiological improvement during the course of TB treatment. Unfortunately, 43 days later, despite the addition of vancomycin to the treatment as well as other supportive measures, the patient died of methicillin-resistant Staphylococcus aureus septicemia.

A total of seven growth cultures were RFLP-typed, with sputum strains exhibiting two genotypic banding patterns with the $10(\mathrm{~B})$ and 13 (A) IS6110 copies and only the "B" pattern in blood. All bands presented the same size and number of copies, except for the three extra-bands in pattern "A" (Figure 1 and Table 1). Analysis of 20 single colonies from blood sub-cultures revealed compartmentalization of the infection due only to the "B" clonal pattern. The same single nucleotide polymorphism in the rpsL gene was observed in all isolates (Lys $88 \rightarrow$ Arg mutation), while for the other genes a wild-type pattern was identified (Table 1).

With regard to the exact match of 10 of 13 bands, this may be a subpopulation with the same clonal origin and thus may be related to the IS6110 transposition as described previously (12). The compartmentalization event has been explained by the presence of both isolates in the initial respiratory infection, with the one more adapted later 
being disseminated $(1,13)$. Our observation of only the " $\mathrm{B}$ " pattern in blood strains may corroborate these previous reports. Garcia de Viedma et al. (1) also suggest that differences in susceptibility to the antimicrobial effects of an extrapulmonary organ may be involved in compartmentalization. In our study, the difference in EMB resistance may have been related to the technical problem, but as the test was repeated twice, this limitation may have been associated with other factors such as the drug's bacteriostatic nature as well as instability and low activity in culture medium. The limitations of genotyping methods to determine the EMB susceptibility pattern have been recently described (8). All of this suggests that the patient was simply harboring a variant of the same strain with small change in genotypic patterns and with the same phenotype and genotypic drug susceptibility profiles, the most adapted of which was disseminated. Previous studies have reported that the presence of variant $M$. tuberculosis strains was more frequent in patients with extrapulmonary and pulmonary TB (12). These investigators did not check for the presence of both variants by examining several colonies isolated from the initial culture, as we did. This allowed us to confirm compartmentalization of one of the variants. Laboratory cross-contamination and partial DNA digestion were ruled out because the other specimens examined resulted in TB-negative cultures, and the digestion procedure was carried out twice under the same conditions. Another result is that the correlation between the DST result and mutation in the genes associated with resistance to INH and RIF failed. Discrepancies in molecular detection related to phenotypic results have been previously documented, including those in Brazilian clinical strains $(14,15)$. Mutation in these strains may have occurred in a different region than that of the target gene or perhaps there were other resistance mechanisms involved. Molecular methods are useful for the rapid detection of most common mutations in MDR cases. However, results should always be confirmed by the appropriate phenotypic method.

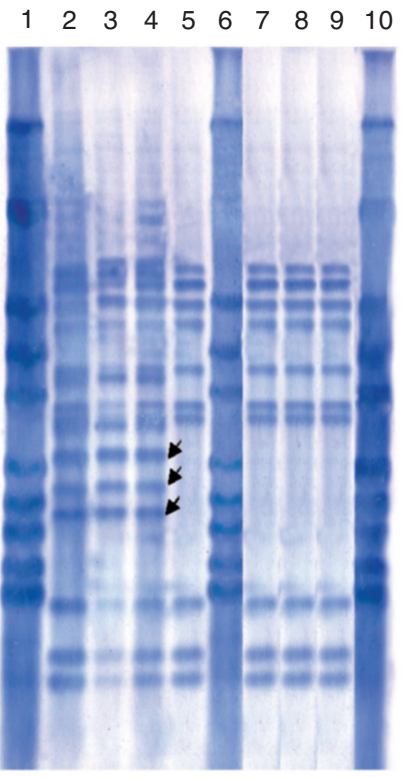

Figure 1. DNA fingerprinting patterns obtained by IS6110-RFLP of multiple MDR Mycobacterium tuberculosis isolates from a patient. Lanes 1, 6, and 10: M. tuberculosis reference strain 14323. Lanes 2, 3, and 4: Sputum (Sp) isolates with pattern A, harboring three extra bands (arrows). Lane 9: Sp isolate with pattern B. Lanes 5, 7, and 8: Blood isolates showing only pattern B.

Table 1. Molecular typing and drug susceptibility (phenotypic and genotypic profile) of multiple blood and sputum isolates from the same patient with pulmonary and disseminated tuberculosis.

\begin{tabular}{|c|c|c|c|c|c|c|c|c|}
\hline \multirow{2}{*}{$\begin{array}{l}\text { Date of clinical } \\
\text { specimen culture }\end{array}$} & \multirow{2}{*}{ ID cultures } & \multirow{2}{*}{$\begin{array}{l}\text { Susceptibility test } \\
\text { (BACTEC 460) }\end{array}$} & \multicolumn{4}{|c|}{ Mutation in genes } & \multirow{2}{*}{$\begin{array}{l}\text { IS6110-RFLP } \\
\text { copy number }\end{array}$} & \multirow{2}{*}{$\begin{array}{l}\text { IS6110-RFLP } \\
\text { pattern }\end{array}$} \\
\hline & & & $r p s L^{1}$ & $\operatorname{inh} A^{2}$ & $r p o B^{3}$ & $e m b B^{4}$ & & \\
\hline \multicolumn{9}{|l|}{ Sputum } \\
\hline $9 / 16 / 2003$ & 1 & $\mathrm{SM}^{\mathrm{R}}, \mathrm{INH}^{\mathrm{R}}, \mathrm{RIF} \mathrm{F}^{\mathrm{R}}, \mathrm{EMB}^{\mathrm{S}}$ & Lys $88 \rightarrow$ Arg & wt & wt & wt & 13 & A \\
\hline $9 / 16 / 2003$ & 2 & $\mathrm{SM}^{\mathrm{R}}$, INH $\mathrm{R}, \mathrm{RIF}^{\mathrm{R}}$, EMB & Lys $88 \rightarrow$ Arg & wt & wt & wt & 13 & A \\
\hline $9 / 16 / 2003$ & 3 & $\mathrm{SM}^{\mathrm{R}}, \mathrm{INH}^{\mathrm{R}}, \mathrm{RIF}$, EMBS & Lys88 $\rightarrow$ Arg & $w t$ & wt & wt & 13 & A \\
\hline $9 / 19 / 2003$ & 7 & $\mathrm{SM}^{\mathrm{R}}, \mathrm{INH}^{\mathrm{R}}, \mathrm{RIF}^{\mathrm{R}}, \mathrm{EMB}$ & Lys $88 \rightarrow$ Arg & wt & wt & wt & 10 & $\mathrm{~B}$ \\
\hline \multicolumn{9}{|l|}{ Blood } \\
\hline $9 / 3 / 2003$ & 4 & $\mathrm{SM}^{\mathrm{R}}, \mathrm{INH}^{\mathrm{R}}, \mathrm{RIF}^{\mathrm{R}}, \mathrm{EMB}^{\mathrm{R}}$ & nd & nd & nd & nd & 10 & B \\
\hline $9 / 3 / 2003$ & 5 & $\mathrm{SM}^{\mathrm{R}}, \mathrm{INH}^{\mathrm{R}}, \mathrm{RIF}^{\mathrm{R}}, \mathrm{EMB}^{\mathrm{R}}$ & Lys $88 \rightarrow$ Arg & wt & wt & wt & 10 & $\mathrm{~B}$ \\
\hline $9 / 3 / 2003$ & 6 & $\mathrm{SM}^{\mathrm{R}}, \mathrm{INH}^{\mathrm{R}}, \mathrm{RIF}^{\mathrm{R}}, \mathrm{EMB}^{\mathrm{R}}$ & Lys $88 \rightarrow$ Arg & wt & wt & wt & 10 & $\mathrm{~B}$ \\
\hline
\end{tabular}

$\mathrm{SM}=$ streptomycin; INH = isoniazid; RIF = rifampicin; EMB = ethambutol; RFLP = restriction fragment length polymorphism; nd = not done; $w t=$ wild type; $R$ = resistant; $S=$ sensitive. Genebank accession number: ${ }^{1}$ X52917, ${ }^{2} \mathrm{U} 41388,{ }^{3} \mathrm{~L} 27989,{ }^{4} \mathrm{U} 68480$. 
The case reported here involved a patient with several risk factors for endogenous reactivation and exogenous re-infection, such as a history of TB 20 years earlier, homelessness, HIV positivity, a previous history of admission to the Emergency Room and to the MDR-TB Reference Center of the hospital, and living in a public shelter in a city with a high TB incidence. Old episode reactivation seems to be unlikely, since at that time the disease was controlled with standard treatment for susceptible MTB. However, as the strain from the old episode was not available for typing we cannot be certain.

In Brazil, especially in Rio de Janeiro, $30 \%$ of the TB cases are diagnosed in hospitals. Public and private hospitals differ in their rates of suspicion and isolation of patients who are considered to be at risk for TB. Emergency Rooms, in general, do not have any TB control program. In a retrospective study carried out to evaluate indicators of TB risk transmission, where $63.5 \%$ of patients were admitted to the hospital through the Emergency Room, in $27.5 \%$ of them the interval between admission and sputum collection exceeded $12 \mathrm{~h}$. This delay in isolation was correlated with diagnosis of TB at admission and lower bacillary load in the sputum (16). In previous Brazilian MDR case studies, there was a significant association with clustering, suggesting that at tertiary care hospitals MDR cases may result from new transmission of primary resistant organisms (17). According to this information, a patient could be infected with one genetic pattern of MDR strain transmitted through the health facilities where he was admitted and the variant pattern found was related to an event of microevolution through IS6110 transposition

\section{References}

1. Garcia de Viedma D, Marin M, Ruiz Serrano MJ, Alcala L, Bouza E. Polyclonal and compartmentalized infection by Mycobacterium tuberculosis in patients with both respiratory and extrarespiratory involvement. J Infect Dis 2003; 187: 695-699.

2. Secretaria e Defesa Civil do Estado do Rio de Janeiro. Saúde - Rio de Janeiro [Update 2007 May 26]. Accessed May 23, 2008.

3. Ministério da Saúde. Controle da tuberculose: uma proposta de integração ensino-serviço. 5th edn. Rio de Janeiro: Fundação Nacional de Saúde. Centro de Referência Prof. Hélio Fraga. Sociedade Brasileira de Pneumologia e Tisiologia; 2002.

4. Telenti A, Imboden P, Marchesi F, Lowrie D, Cole S, Colston $\mathrm{MJ}$, et al. Detection of rifampicin-resistance mutations in Mycobacterium tuberculosis. Lancet 1993; 341: 647-650.

5. Miller LP, Crawford JT, Shinnick TM. The rpoB gene of Mycobacterium tuberculosis. Antimicrob Agents Chemother over time. Such event has been described previously in serial patient isolates and most of the variant patterns $(68 \%)$, with a gain or loss of 1 to 3 copies of IS6110, were taken 0-29 days after the initial isolate (12).

Although HIV infection has not been conclusively proven as an independent risk factor for drug resistance, MDR-TB outbreaks in HIV scenarios and high mortality rates in people with MDR-TB/HIV co-infection justify routine DST in all HIV/TB-infected patients. In Brazil all cases of TB retreatment are indicated for DST.

The case reported here presented an event of infection in which RFLP fingerprint resulted in the detection of variant strains, with discrepancy among MDR phenotypes together with a genotype mutation profile, suggesting serious implications with regard to treatment administration exclusively based on molecular methods, and calling attention to the fact that more effective control strategies against the emergence of MDR strains must be implemented by the TB control program to prevent transmission of M. tuberculosis-MDR strains at health facilities in Rio de Janeiro.

\section{Acknowledgments}

We thank Glenn P. Morlock (Center for Disease Control, Atlanta, GO, USA) for DNA sequencing and synthesis of oligonucleotide primers and Anna Grazia Marsico (HU/ UFRJ, Rio de Janeiro, RJ, Brazil) for re-testing the susceptibility pattern of the strains. English was reviewed and revised by Mitchell Raymond Lisbon (Chicago, IL, USA).

1994; 38: 805-811.

6. Honore N, Cole ST. Streptomycin resistance in mycobacteria. Antimicrob Agents Chemother 1994; 38: 238-242.

7. Abbadi S, Rashed HG, Morlock GP, Woodley CL, EI Shanawy O, Cooksey RC. Characterization of IS6110 restriction fragment length polymorphism patterns and mechanisms of antimicrobial resistance for multidrug-resistant isolates of Mycobacterium tuberculosis from a major reference hospital in Assiut, Egypt. J Clin Microbiol 2001; 39: 2330-2334

8. Hazbon $\mathrm{MH}$, Bobadilla del Valle M, Guerrero MI, VarmaBasil M, Filliol I, Cavatore M, et al. Role of embB codon 306 mutations in Mycobacterium tuberculosis revisited: a novel association with broad drug resistance and IS6110 clustering rather than ethambutol resistance. Antimicrob Agents Chemother 2005; 49: 3794-3802.

9. van Embden JD, Cave MD, Crawford JT, Dale JW, Eisenach KD, Gicquel B, et al. Strain identification of Mycobacterium 
tuberculosis by DNA fingerprinting: recommendations for a standardized methodology. J Clin Microbiol 1993; 31: 406409.

10. Saad MH, Fonseca LD, Ferrazoli L, Fandinho F, Palaci M, Grinsztejn B, et al. IS1245 genotypic analysis of Mycobacterium avium isolates from patients in Brazil. Int $J$ Infect Dis 1999; 3: 192-196.

11. Ministério da Saúde FNdS. Tuberculose - guia de vigilância epidemiológica. 1st edn. Brasília: Assessoria de Comunicação e Educação em Saúde (ASCOM); 2002.

12. de Boer AS, Borgdorff MW, de Haas PE, Nagelkerke NJ, van Embden JD, van Soolingen D. Analysis of rate of change of IS6110 RFLP patterns of Mycobacterium tuberculosis based on serial patient isolates. $J$ Infect Dis 1999; 180: 1238-1244.

13. Braden CR, Morlock GP, Woodley CL, Johnson KR, Colombel AC, Cave MD, et al. Simultaneous infection with multiple strains of Mycobacterium tuberculosis. Clin Infect Dis 2001; 33: e42-e47.

14. Bartfai Z, Somoskovi A, Kodmon C, Szabo N, Puskas E,
Kosztolanyi L, et al. Molecular characterization of rifampinresistant isolates of Mycobacterium tuberculosis from Hungary by DNA sequencing and the line probe assay. J Clin Microbiol 2001; 39: 3736-3739.

15. Freixo MI, Caldas PC, Said A, Martins F, Brito RC, Fonseca LS, et al. Antimicrobial susceptibility determined by the $E$ test, Lowenstein-Jensen proportion, and DNA sequencing methods among Mycobacterium tuberculosis isolates discrepancies, preliminary results. Mem Inst Oswaldo Cruz 2004; 99: 107-110.

16. Resende MR, Sinkoc VM, Garcia MT, Moraes EO, Kritski $\mathrm{AL}$, Papaiordanou PMO. Indicators related to delays in diagnosis and in implementation of measures to control airborne infection among patients with pulmonary tuberculosis in tertiary-care hospital. J Bras Pneumol 2005; 31: 225-230.

17. Fandinho FC, Kritski AL, Hofer C, Junior Conde H, Ferreira $\mathrm{RM}$, Saad MH, et al. RFLP patterns and risk factors for recent tuberculosis transmission among hospitalized tuberculosis patients in Rio de Janeiro, Brazil. Trans $R$ Soc Trop Med Hyg 2000; 94: 271-275. 